\title{
The Influence of Perineural Invation on the Prognosis of Patients with Gastric Cancer after Radical Operation_A Retrospective Study
}

\author{
Houxin Zhu ${ }^{1,2}$, Lin Sun ${ }^{3}$, Tuo Shi $^{1,2}$
}

\author{
${ }^{1}$ Department of General surgery, Affiliated Hospital of Qingdao University, Shandong, Qingdao, 266003 \\ ${ }^{2}$ Department of medicine, Qingdao university, Shandong, Qingdao, 266003 \\ ${ }^{3}$ Shandong University, Shandong, Jinan, 250000
}

\begin{abstract}
Objective to investigate the influence of perineural invation (PNI) on the prognosis of patients with gastric cancer after radical resection. Method: we collected 278 patients in Affiliated Hospital of Qingdao University with gastric cancer after radical operation and to analysis. The specimens of gastric cancer were stained with hematoxylin and eosin (HE), when the tumor cells were infiltrated the membrane or the nerve bundles, it would be defined as PNI positive. To analyzed the relationship between PNI and other clinic-pathological features and the influence of prognosis. Results: The 5-year overall survival of the patients with PNI were lower than those of the patients with negative perineural infiltration $(26.45 \%$ vs $43.72 \%, P<0.05)$. Multivariate analysis showed that PNI was an independent factor affecting the prognosis of the patients $(\mathrm{p}=0.023$, HR $=0.182,95 \mathrm{CI}: 0.042-0.793)$. The rate of PNI was $30.57 \%(85 / 278)$. PNI positive was related to TNM stage, tumor differentiation, vascular and lymphatic invasion and Lauren classification $(\mathrm{p}<0.05)$., but the size of tumor, the location of tumor and Borrmann classification were not significant $(\mathrm{p}>0.05)$. Conclusion: patients with PNI positive with lower rate of survival. PNI positive is related to the progression of gastric cancer.
\end{abstract}

Keywords: Gastric Cancer, Perineural Infiltration, Radical Operation, Prognosis

Gastric cancer is the third tumor-related mortality in the world. In China, gastric cancer had been ranked the third place in the causes of tumor-related deaths[1, 2]. The early diagnosis rate of gastric cancer in China is relatively low and less than $20 \%$. Most of the patients were treated with locally advanced cancer(T2-4N2-3M0) when they were diagnosed at the first time[3]. Radical surgery is still the main method for the treatment of gastric cancer at present, after operation, there were some factors which is associated with poor prognosis of patients [4, 5]. PNI is a process which the tumor invade the bundles or bundles of nerves and encircle the nerves. PNI existed in pancreatic, biliary, prostate and head and neck malignancies, it is reported that PNI would be an important means of local diffusion of tumors and with a poor prognosis[6].

\section{Materials and methods}

(1) Patients data

We collected the patients from June 2009 to June 2014 from our tumor database, 278 patients with gastric cancer were treated with radical surgery in Affiliated Hospital of Qingdao University. All patients did not taken radiotherapy and chemotherapy before the operation, and it is proved that there is no distant metastasis by Imaging data, such as CT, enhanced CT, endoscopic ultrasonography. Follow-up was carried out by telephone or outpatient after the operation, the deadline of follow-up time was June 2018, the rate of follow-up was $88.5 \%$. TNM staging was based on the seventh edition staging criteria of the American Cancer Staging Committee(AJCC).

\section{(2) Method}

The tissues of patients with gastric cancer were collected and fixed with $10 \%$ formaldehyde., then were embedded with paraffin, the sections were sliced with $5 \mu \mathrm{m}$ thickness and stained with hematoxylin eosin. When the cancer cells infiltrate into the nerve bundles, it can be judged to be PNI positive. After that, we evaluate the relationship between PNI positive and other clinic-pathological features of gastric cancer, and to analyze the influence of PNI on the prognosis of gastric cancer patients. After checked, patients with positive PNI was listed as trial group, patients with negative PNI was listed as control group.

\section{(3) Surgery}

Surgical procedures were performed in accordance 
The Influence of Perineural Invation on the Prognosis of Patients with Gastric Cancer after Radical Operation _A Retrospective Study

with the 2014 Japanese gastric Cancer treatment guidelines (ver.4). Radical total gastrectomy, the whole stomach was removed, with the proximal line of division through the distal esophagus, and the distal line of division through the proximal duodenum. The procedure for a radical subtotal distal gastrectomy was the same, but a small, viable gastric remnant was left intact. In both procedures, the resection lines had to be at least $5 \mathrm{~cm}$ from the edge of the macroscopic tumor, when this rule could not be observed, frozen section was used to examine the resection margin. Lymph nodes resections strictly adhered to Standard D2 resection.

\section{(4) Data collection}

The clinic-pathological data of the patients were collected, identified, recorded and stored in the gastrointestinal tumor database two independent researchers.

\section{(5)Statistical method}

$\chi^{2}$ test was used for the counting data, the survival rate was calculated by kaplan Meier method, the survival rate was compared by log-rank test, and the cox proportional risk model was used for multivariate survival analysis. SPSS 24.0 was used to deal with data, $P<0.05$ was statistically significant.

\section{Result}

(1) Clinic-pathological characteristics

The relationship between PNI and other clinic-pathological features: among 278 patients, 85 cases were positive for PNI, the positive rate was $30.57 \%$. The positive rate of PNI was related to TNM stage, tumor differentiation, vascular and lymphatic invasion, Lauren classification $(P<0.05)$. Tumor size, tumor location and Borrmann classification were not correlated $(P>0.05)$, as is shown in table 1 .

\begin{tabular}{|c|c|c|c|c|}
\hline & $\begin{array}{l}\text { Trial group } \\
\text { (PNI+) }\end{array}$ & $\begin{array}{l}\text { Control group } \\
\text { (PNI-) }\end{array}$ & $\chi^{2 \text { Value }}$ & P Value \\
\hline Age & & & 1.005 & 0.325 \\
\hline$>60$ & 65 & 99 & & \\
\hline$<60$ & 20 & 94 & & \\
\hline Sex & & & 0.124 & 0.721 \\
\hline Male & 61 & 140 & & \\
\hline Female & 24 & 53 & & \\
\hline Size of tumor(cm) & & & 0.227 & 0.628 \\
\hline$\geq 5$ & 55 & 94 & & \\
\hline$<5$ & 30 & 99 & & \\
\hline Location & & & 0.856 & 0.867 \\
\hline Upper1/3 & 16 & 28 & & \\
\hline Middle1/3 & 8 & 19 & & \\
\hline Lower $1 / 3$ & 49 & 126 & & \\
\hline$\geq 2 / 3$ & 12 & 20 & & \\
\hline $\begin{array}{l}\text { Lauren } \\
\text { classification }\end{array}$ & & & 6.472 & $0.010^{*}$ \\
\hline intestinal & 37 & 66 & & \\
\hline Diffuse & 47 & 127 & & \\
\hline $\begin{array}{l}\text { Borrmann } \\
\text { classification }\end{array}$ & & & 0.598 & 0.258 \\
\hline I-II & 39 & 110 & & \\
\hline III-IV & 46 & 83 & & \\
\hline TNM Stage & & & 24.487 & $<0.001^{*}$ \\
\hline
\end{tabular}


The Influence of Perineural Invation on the Prognosis of Patients with Gastric Cancer after Radical Operation _A Retrospective Study

\begin{tabular}{|c|c|c|c|c|}
\hline Istage & 2 & 13 & & \\
\hline II stage & 9 & 75 & & \\
\hline III stage & 74 & 105 & & \\
\hline $\begin{array}{l}\text { Tumor } \\
\text { differentiation }\end{array}$ & & & 10.268 & 0.006 \\
\hline G1 & 0 & 0 & & \\
\hline G2 & 26 & 57 & & \\
\hline G3 & 59 & 136 & & \\
\hline vascular invasion & & & 19.789 & $<0.001 *$ \\
\hline Yes & 29 & 58 & & \\
\hline No & 56 & 135 & & \\
\hline $\begin{array}{l}\text { lymphatic } \\
\text { invasion }\end{array}$ & & & 18.425 & $<0.001^{*}$ \\
\hline Yes & 31 & 65 & & \\
\hline No & 54 & 128 & & \\
\hline
\end{tabular}

\section{(2) Influence of PNI on survival}

As shown in figure 1, the overall survival of the trial group was significantly higher than that in the control group. The rate of overall survival was $35.45 \%$ and
$55.72 \%$ in these two groups, $(\mathrm{P}<0.05)$. Multivariate analysis showed that PNI was an independent factor affecting the prognosis of the patients $(\mathrm{p}=0.023, \mathrm{HR}=$ 0.182, 95CI: $0.042-0.793)$, as is shown in Table 2.

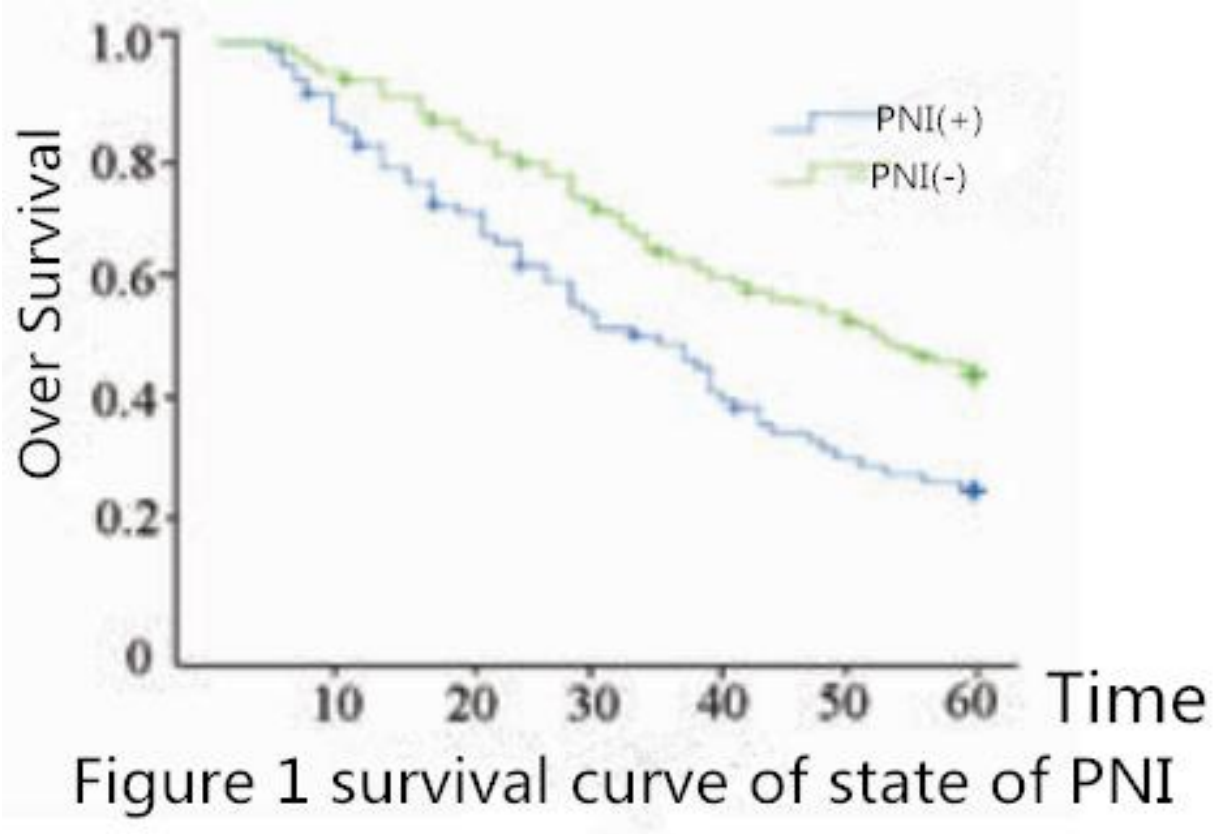


The Influence of Perineural Invation on the Prognosis of Patients with Gastric Cancer after Radical Operation _A Retrospective Study

\begin{tabular}{|l|l|l|l|}
\hline \multicolumn{5}{|c|}{ Table 2 COX multivariate analysis of overall survival } \\
\hline PNI & HR & $95 \%$ CI & $P$ Value \\
\hline+ & & & $0.024^{*}$ \\
\hline- & 0.172 & $0.049-0.783$ & \\
\hline T stage & 1.000 & & \\
\hline T2 & & & $0.020^{*}$ \\
\hline T3 & 0.928 & $0.123-6.952$ & 0.008 \\
\hline T4 & 0.349 & $0.038-3.250$ & 0.107 \\
\hline N stage & 1.000 & & \\
\hline N0 & & & $0.002 *$ \\
\hline N1 & 0.475 & $0.258-0.687$ & 0.001 \\
\hline N2 & 0.555 & $0.327-0.761$ & 0.003 \\
\hline N3 & 0.611 & $0.476-1.095$ & 0.114 \\
\hline & 1.000 & & \\
\hline
\end{tabular}

\section{Discussion}

Gastric cancer is one of the most common malignant tumors in the world. Two thirds of patients were found in developing countries, especially in China and other Southeast Asian countries. The mortality rate is high, although the 5-year survival rate of early gastric cancer can reach more than $90 \%$. However, the overall 5-year survival rate was about $30 \%-50 \%$. The early patients were not screened for lack of symptoms. Once diagnosed, the prognosis of patients with gastric cancer after operation was advanced. Surgery is recognized as the best treatment method at present. At the same time, the prognostic factors were analyzed, which is helpful to understand the high risk population with the possibility of recurrence after operation, and to provide a strong basis for clinical selection of more scientific and reasonable treatment plan. It is of great significance to improve the prognosis of gastric cancer after operation.

It has been reported that PNI is an important diffusion pathway in pancreatic, biliary and colorectal tumors. The positive rate of PNI was about $20 \%$ in colorectal cancer and $70 \% \sim 100 \%$ in pancreatic carcinoma and biliary tract tumor. It is reported that the rate of positive PNI was $73.0 \%$ in 287 patients with gastric cancer. There were significant differences in the depth of tumor invasion and lymph node metastasis between PNI positive and negative patients $(\mathrm{P}<0.001)$.Some people found that the positive rate of PNI was closely related to tumor size, degree of differentiation, lymph node metastasis, depth of tumor invasion, vascular and lymphatic canal invasion, and TNM stage. The positive rate of PNI was also higher with the progression of tumor. There was also similar conclusion in other research. In our study, the rate of positive PNI was $30.57 \%$. The positive rate of PNI was correlated with
TNM stage, tumor differentiation, vascular and lymphatic invasion and Lauren classification $(\mathrm{p}<0$. $05)$. With the increase of the depth of tumor invasion, the progress of clinic-pathologic stage, the decrease of tumor differentiation, the positive rate of PNI also increased significantly $(\mathrm{p}<0.01)$. The incidence of vascular and lymphatic invasion in PNI positive patients was significantly higher than that in PNI negative patients. Some scholars found that with the increase of lymph node metastasis would increase the positive rate of PNI. The rate of positive PNI in patients with lymphocytic metastasis (N1-3) was significantly higher than that in patients without lymphatic node metastasis (N0). However, there was no significant difference in the positive rate of PNI among the patients with lymph node metastasis $(\mathrm{p}>0$. 05) and the positive rate did not increase with the increase of the number of lymph node metastasis. The results suggest that the positive rate of PNI is related to lymph node metastasis, but it may not be related to the specific number of lymph node metastasis.

Up to now, the mechanism of PNI has not been fully clarified. It was suggested that the occurrence of PNI in pancreatic cancer may be related to the anatomic position of the celiac plexus and pancreas. Kameda et al. think that when tumor cells invade the membranes of the nerve tract, they call them PNI, they also found that the number of layers of the fascicular membrane of the terminal nerve was significantly less than that of the central nervous system. As a result, tumor cells are more likely to invade the terminal nerve around the tumor through the fascicular membrane. PNI was not only related to its anatomical specificity, more importantly, tumor cells can recognize and invade neural tissue by secreting neuronal adhesion molecule (NCAM). They also found that the occurrence of PNI 
is closely related to the invasion of blood vessels and lymphatic vessels, PNI is not only the result of direct infiltration of tumor cells, but also may be related to the invasion of blood vessels and lymphatic vessels around the nerve by tumor cells. However, it is showed that PNI is an independent pathway of metastasis because the perineural space is different from the lymphatic canal in anatomical and microstructural aspects. In our study it is showed that the positive rate of PNI was closely related to the invasion of blood vessels and lymphatic vessels, which may be one of the reasons for the high incidence of PNI[7].

There is also no consensus on the impact of PNI on the prognosis of gastric cancer. Zhou et al. suggest that PNI would speed up the progression of gastric cancer, but PNI is not an independent prognostic factor. Some people studied 1372 patients with gastric cancer after radical resection. They were the first found and reported that PNI was an independent prognostic factor affecting the overall survival rate of patients. In recent years, we found that the prognosis of PNI positive patients was significantly worse than that of PNI negative patients. They also confirmed that PNI was an independent prognostic factor. The 5-year overall survival rate and mean survival time of PNI positive patients were significantly lower than those of PNI negative patients $(P=0.001)$. PNI was an independent prognostic factor $(P=0.023, \mathrm{HR}=0.182,95 \% \mathrm{CI}$ 0.042-0.793). Therefore, we believe that PNI positive is one of the indicators of poor prognosis in patients with gastric cancer.

Some people found that PNI positive was associated with peritoneal metastasis of gastric cancer, but not with hepatic metastasis of gastric cancer. Zhou et al. also found that PNI was associated with recurrence and metastasis of gastric cancer. Because the data of this group we did not collected patients with distant metastasis and all patients underwent radical surgical treatment, there is no discussion was made on the relationship between PNI and recurrence and metastasis of gastric cancer[8].

In a word, the incidence of PNI in patients with gastric cancer is higher. PNI was closely related to TNM stage, tumor differentiation, vascular and lymphatic canal invasion, and Lauren classification. The positive rate of PNI increased with the progression of gastric cancer. PNI is an independent factor affected the prognosis of gastric cancer patients. Therefore, for patients with gastric cancer after radical resection, positive PNI may become an important new indicator for poor prognosis, and provide a new strategy and method for the diagnosis and treatment of gastric cancer.

\section{Reference}

1. Sakuramoto, S., et al., Adjuvant chemotherapy for gastric cancer with S-1, an oral fluoropyrimidine. N Engl J Med, 2007. 357(18): p. 1810-20.
2. Strong, V.E., et al., Comparison of gastric cancer survival following R0 resection in the United States and Korea using an internationally validated nomogram. Ann Surg, 2010. 251(4): p. 640-6.

3. Yazici, O., et al., The effect of the gastrectomy on survival in patients with metastatic gastric cancer: a study of ASMO. Future Oncol, 2016. 12(3): p. 343-54.

4. Nakajima, T., Gastric cancer treatment guidelines in Japan. Gastric Cancer, 2002. 5(1): p. 1-5.

5. Huang, J.Y., et al., The prognostic impact of occult lymph node metastasis in node-negative gastric cancer: a systematic review and meta-analysis. Ann Surg Oncol, 2013. 20(12): p. 3927-34.

6. Zhang, X.F., et al., Surgical treatment and prognosis of gastric cancer in 2,613 patients. World J Gastroenterol, 2004. 10(23): p. 3405-8.

7. An, J.Y., et al., Pathologic and oncologic outcomes in locally advanced gastric cancer with neoadjuvant chemotherapy or chemoradiotherapy. Yonsei Med J, 2013. 54(4): p. 888-94.

8. Li, P., et al., Vascular invasion as an independent predictor of poor prognosis in nonmetastatic gastric cancer after curative resection. Int J Clin Exp Pathol, 2015. 8(4): p. 3910-8. 